Cahiers $d u$ MONDE RUSSE

\section{Cahiers du monde russe}

Russie - Empire russe - Union soviétique et États indépendants

$58 / 4 \mid 2017$

Varia

\title{
Irina Paperno, Tchernychevski et l'âge du réalisme : Essai de sémiotique des comportements
}

\section{Gábor T. Rittersporn}

\section{(2) OpenEdition}

Journals

Édition électronique

URL : http://journals.openedition.org/monderusse/10198

DOI : $10.4000 /$ monderusse. 10198

ISSN : $1777-5388$

Éditeur

Éditions de l'EHESS

\section{Édition imprimée}

Date de publication : 1 octobre 2017

Pagination : 754-757

ISBN : 978-2-7132-2698-4

ISSN : $1252-6576$

\section{Référence électronique}

Gábor T. Rittersporn, «Irina Paperno, Tchernychevski et l'âge du réalisme : Essai de sémiotique des comportements », Cahiers du monde russe [En ligne], 58/4 | 2017, mis en ligne le 01 octobre 2017, consulté le 06 janvier 2021. URL : http://journals.openedition.org/monderusse/10198 ; DOI : https:// doi.org/10.4000/monderusse.10198 


\section{Irina PAPERNO}

\section{Tchernychevski et l'âge du réalisme}

\section{Essai de sémiotique des comportements}

Lyon : ENS Éditions, 2017, 302 p. (trad. de l'anglais par Aurélien Langlois)

L'idée que nos concepts sont des constructions n'a rien de surprenant de nos jours, mais tout le monde n'en est pas conscient. Les réalistes russes du XIX ${ }^{\mathrm{e}}$ siècle n'échappaient pas à la règle : ils construisaient la réalité dont ils se réclamaient, sans soupçonner qu'ils la composaient selon leurs idées reçues. Ils ont marqué leur époque et leur influence s'est fait sentir jusqu'aux dernières décennies de l'ère soviétique. Ces réalistes n'étaient pas que des hommes de lettres qu'on associe à la tendance esthétique qualifiée de réalisme.

Irina Paperno saisit une période de l'histoire russe où le réalisme, dans un sens large du terme, était une vision du monde, ainsi que le fondement d'un certain mode de vie et de son apprentissage. Elle examine le rôle que Nikolaj Černyševskij, figure phare de l'époque, a joué en tant qu'apprenti, éducateur et propagandiste. Elle explore ainsi les leitmotive du courant et la dialectique du réel et de l'idéal dans son imaginaire et celui de ses compagnons d'âme.

L'âge du réalisme russe était un peu moins réaliste qu'il n'y paraît au premier coup d'œil. Les efforts de construire un réel et de s'y adapter n'étaient pas tout à fait étrangers aux exercices romantiques de subordination de sa personne à de nobles idéaux. Toutefois, les idéaux des héros de Paperno étaient incomparablement plus liés à l'aspect concret du monde que ceux des romantiques. Ils les distillaient d'une réalité construite à partir du rationalisme et en fonction des méthodes et des découvertes des sciences naturelles et sociales de leur temps. Paperno ne se laisse pas piéger par la tentation de déconstruire ce réel pour expliquer ce qui en semble véridique ou faux du point de vue d'une réalité qu'on peut construire aujourd'hui. Elle s'y intéresse dans la perspective de l'existence que ses acteurs jugeaient authentique, dans celle de leurs tentatives de la vivre et dans les textes que Černyševskij a laissés. Les réalistes étaient à la recherche d'une authenticité qui n'était pas très 
éloignée de la volonté romantique de servir l'humanité. Il s'y ajoutait une détermination d'avancer ce qu'ils comprenaient comme la marche scientifiquement démontrée du progrès dans leur quotidien, dans la société et dans l'histoire.

Comme les romantiques, les réalistes s'inspiraient de modèles littéraires pour forger leur personnalité. Paperno met en exergue de son livre une citation de Turgenev qui a écrit qu'à la fin du XVIII ${ }^{\mathrm{e}}$ siècle, les Allemands avaient quelque chose du Werther et de la Charlotte goethéens et, plus tard, de ce qu'ils prenaient pour les brigands de Schiller, ajoutant qu'après 1862, presque tous les jeunes Russes sortaient du Que faire? de Černyševskij avec quelques traits de Bazarov, protagoniste de son propre Pères et Fils, écrit au début des années 1860. Les principaux blocs de construction du réel des réalistes étaient donc redevables à Černyševskij. Malgré son maigre mérite littéraire, son roman allait captiver plusieurs générations. Lénine avouait que le livre avait radicalement changé sa vie.

Černyševskij était de ces roturiers qui, à partir des années 1840 , accédèrent de plus en plus souvent aux professions intellectuelles. À la différence de la plupart de leurs prédécesseurs, qui étaient issus de milieux nobles, ils étaient des raznočincy, des «gens de rangs variés », selon l'excellente trouvaille du traducteur Aurélien Langlois. Ils commençaient de zéro dans leur nouvel environnement et ils montaient rarement très haut dans la hiérarchie sociale. En revanche, ils créaient leur propre sphère sociale avec ses valeurs, ses codes culturels et ses préceptes sur la vie vertueuse.

Černyševskij était à la fois produit et producteur de ce milieu, de ses normes et de ses manières d'exister. À l'instar des autres individus issus de rangs variés, il éprouvait les mêmes difficultés de se sentir à l'aise dans la société distinguée et le même besoin de trouver la compagnie de gens de statut social comparable au sien, y compris en cherchant la femme idéale. Matérialiste dans tous les domaines de la pensée comme ses compères, il croyait important de pouvoir localiser dans l'organisme humain et dans son propre corps, la source des émotions et des sentiments élevés. En souscrivant aux convictions de son milieu, pour lui les termes comme celui du « cœur » relevaient du concret, même dans les cas où ils s'appliquaient dans un sens métaphorique.

Un domaine privilégié de l'existence authentique des réalistes était le mariage avec tous les méandres de l'itinéraire conjugal. Dans un premier temps, il s'agissait d'entamer l'alliance en établissant une relation d'égalité entre les partenaires, chose rare selon les conventions prédominantes de l'époque. À ce stade, le futur et le jeune mari étaient légèrement plus égaux que la fiancée et l'épouse, car c'est à eux qu'incombaient la tâche d'éduquer la femme dans l'esprit des temps nouveaux. Si le travail pédagogique de Černyševskij ne fut pas couronné de succès en ce qui concerne l'engagement social et politique de sa conjointe, son enseignement sur l'égalité porta vite ses fruits : sa femme menait une vie mondaine et quelque peu frivole, qu'un réaliste se devait d'accepter. L'union devait constituer un espace de liberté que les convenances de la société respectable n'étaient pas censées restreindre.

En tant que bons hégéliens, Černyševskij et les siens se pliaient sans rechigner à la rationalité de leurs réalités. En tant que mauvais hégéliens, ils ne se demandaient 
pas si, ni à quel point, la logique interne de leurs constructions et leurs constructions en tant que telles relevaient de la nécessité. Les éléments qu'ils choisissaient ou fabriquaient n'étaient pas les seuls matériaux imaginables et pensables pour édifier leur univers, mais d'autres éléments avaient de bonnes chances d'impliquer des contraintes auxquelles ils voulaient échapper à tout prix. Černyševskij n'était pas à court d'arguments scientifiques pour justifier l'état de ses propres affaires dans son mariage. Il en allait de même pour ses frères et sœurs réalistes qui n'éprouvaient pas grande difficulté à vivre en alliance à trois et y trouver leur bonheur. Les temps romantiques où les ménages de la sorte furent bouleversés par des déchirements passionnés n'étaient pourtant pas loin. Que faire? a convaincu les adeptes du réalisme que le fondement de la vraie union amoureuse était l'ambition partagée de comprendre et changer le monde.

Le roman servait de modèle pour une jeunesse (et même pour un public moins jeune) en quête de modes de vie sortant du carcan d'une société autoritaire, patriarcale, égoïste et hypocrite. Il était un compendium des idées progressistes russes de son âge et un manuel, même pour ceux qui ne voulaient pas expérimenter de nouvelles formes de coexistence maritale. Il faisait miroiter le projet du bonheur collectif d'êtres égaux, prêts à œuvrer pour le bien commun et à supporter toutes les épreuves en accomplissant leur devoir. Le roman n'était pas tout à fait utopique. Son action se déroulait dans le présent et il suggérait que le lecteur pouvait se lancer dans l'aventure immédiatement, pourvu qu'il soit destiné à développer sa personnalité pour devenir à même de s'atteler aux tâches du réalisme et digne de s'intégrer dans une communauté créatrice d'un avenir heureux.

Paperno convainc le lecteur que la structure du livre est plus complexe qu'elle n'est habituellement comprise. Le roman suit un schéma dialectique. Les qualités des divers personnages forment des contradictions, les figures ont des doubles et leurs antagonismes trouvent leur résolution dans leur dépassement hégélien (dans la Aufhebung), ce qui mène à une véritable transfiguration des protagonistes. L'écrivain fait apparaître un monde d'apparences, celui des interprétations illusoires de leur vécu par les acteurs. Černyševskij le déconstruit pour représenter ce qu'il veut exposer comme réalité. Il montre que celle-ci se révèle grâce à l'intellect du réaliste, sans que l'idée l'effleure qu'il préconise par là même sa construction.

De leur propre aveu Que faire? était un catéchisme pour ses admirateurs. Ils étaient prêts à y voir la source «d'une nouvelle foi » ou « l'Évangile du socialisme ». Paperno remonte aux origines chrétiennes du credo socialiste des années 1830 et 1840 en France, en Allemagne et en Angleterre. D’une part, elle montre comment ce credo rédempteur apparaît dans la vision du monde des personnages clés du roman, de l'autre, elle met en évidence, dans le trajet intellectuel de Černyševskij, l'importance de son éducation de séminariste, qui aboutit à une synthèse du dogme orthodoxe exploré dans le journal intime de sa jeunesse et de ce qu'il appellera plus tard rationalisme. Paperno dégage une véritable théologie de son œuvre nourrie de symbolisme chrétien, pleine d'allusions bibliques et culminant dans une vision prophétique d'un paradis habité d'humains épanouis dans le travail et dans l'amour libre. 
Les anciens séminaristes et les individus issus de familles de prêtres et de milieux ecclésiastiques étaient hautement visibles dans la subculture radicale. Paperno cite Myškin, l'idiot de Dostoevskij, qui dit qu'ils étaient convertis à l'athéisme « comme à une foi nouvelle ». Elle n'est pas tout à fait certaine si, ni dans quelle mesure, ses acteurs sont des athées et saisissent les ingrédients de la nouvelle foi. Il s'agit entre autres d'une quasi-théologie fondée sur un déterminisme qui exclut le libre arbitre et envisage une rationalité utilitariste inscrite dans le réel de ses adeptes. Ceux-ci vouaient à Černyševskij une vénération qui n'était pas loin de la ferveur religieuse. L'écrivain a achevé son roman après son arrestation, à la forteresse Pierre-et-Paul de Saint-Pétersbourg. Il avait trente-quatre ans et il allait passer les trente-sept qui lui restaient au bagne et en exil. Le régime a contribué à la sanctification virtuelle de sa figure.

Paperno convoque un éventail impressionnant de sources et de travaux savants. Elle fait preuve d'une connaissance profonde des origines et des implications philosophiques et théologiques du réel de Černyševskij et de ses contemporains. La monographie de Paperno n'est pas sans affinités avec la sémiotique des cultures et des modes de vie de Iurij Lotman, mais les dimensions théoriques de son étude dépassent largement celles du maître de Tallinn. Les continuités, les transformations et les ruptures des visions du monde et des comportements qu'elle étudie se déploient dans un espace chronologique dont l'étendue coupe le souffle. Elle place l'âge du réalisme dans la perspective historique des idées et des mentalités dont l'horizon commence dans l'antiquité et finit au-delà de l'époque qu'elle explore.

La première édition du livre remonte à 1988. Il avait été publié en anglais et il vient de paraître en français. Le (re)lecteur d'aujourd'hui voit mieux qu'il y a trente ans l'aspect novateur et pionnier de la démarche de l'auteur. Et trente ans après, l'appréciation est toujours d'actualité. L'analyse sémiotique de la culture russe n'a guère avancé depuis les années 1980 et la première édition de l'ouvrage de Paperno. On se demande même si Paperno n'avait pas déjà mis en évidence des possibilités de l'analyse sémiotique de la culture russe qui ne se sont révélées que depuis ces années quatre-vingt du siècle dernier. Autant dire que le public français reçoit une nouveauté grâce à l'admirable traduction d'Aurélien Langlois.

Gábor T. Rittersporn

Centre national de la recherche scientifique 